\title{
THEORETICAL STUDY OF THE EFFECT OF PROBE SHAPE ON ADHESION FORCE BETWEEN PROBE AND SUBSTRATE IN ATOMIC FORCE MICROSCOPE EXPERIMENT
}

\author{
Li Yang, Junhui Hu, Linguiang Kong \\ College of Physics Science and Technology, Guangxi Normal University, Guilin, China \\ e-mail: hujh@mailbox.gxnu.edu.cn.
}

\begin{abstract}
The quantitative description of adhesion force dependence on the probe shapes are of importance in many scientific and industrial fields. In order to elucidate how the adhesion force varied with the probe shape in atomic force microscope manipulation experiment, we performed a theoretical study of the influences of the probe shape (the sphere and parabolic probe) on the adhesion force at different humidity. We found that the combined action of the triple point and the Kelvin radius guiding the trend of the adhesion force, and these two fundamental parameters are closely related to the probe shape. Whilst, the theoretical results demonstrate that the adhesion force is in good agreement with the experiment data if the van der Waals force is taken into account.
\end{abstract}

Keywords: probe shape, adhesion force, van der Waals force, capillary force, relatively humidity, liquid bridge

\section{Introduction}

The Atomic Force Microscope (AFM) has a great impact on various areas such as nano metrology (Eastman and Zhu, 1996), materials science (Thundat et al., 1993a), surface science and biology (Binnig and Quate, 1986). It is a versatile tool for studying nanomaterials properties such as friction and adhesion forces (Werf et al., 1994). The quality of image obtained from an AFM is greatly dependent both on the probe shape and physical property, especially geometry and dimension of the probe end (Thomas et al., 1995). The chemical and physical parameters of the probe significantly affect AFM measurements and can reduce resolution Thundat et al., 1993ab). Choosing the correct probe is a crucial part of working on biological samples.

Currently, there are many publications devoted to the problem of probe geometries, and different models have been established to investigate the adhesion between objects with different shapes and a solid plane (Butt and Kappl, 2009; Chen and Soh, 2008; Tabrizi et al., 2006, 2008). Most of the previous works focused on the capillary force. Tabrizi et al. (2008) numerically calculated the capillary force for a sphere, cone and a flat cylinder in contact with a planar surface. Their model and experiments showed that changes in tip geometry on the sub-10-nm length scale can completely change the adhesion force versus humidity curves. Chen and Soh (2008) studied the influence of the indenter shapes (conical, spherical and truncated conical with a spherical end) on the magnitude of the capillary force in micro-electro-mechanical systems. Different dependences of the capillary force on the indenter shapes and geometric parameters were observed. Butt and Kappl (2009) calculated the capillary force with three different probe shapes, the sphere, cone and cylinder in perfectly wetted surfaces. They noticed that the capillary force and the liquid bridge rupture distance can change fundamentally with different probe shapes.

It has already been known that the adhesion force (including van der Waals force, capillary force and electrostatic force) (Quyang et al., 2001) significantly changes with the different probe 
shape in the AFM experiment, but details of how the tip shape influences the adhesion force have not been clearly understood. Therefore, a perspective for exhaustive analysis of key parameters is necessary.

In the present paper, we simulated the adhesion force for the sphere probe and the parabolic probe on a substrate in an atomic force microscope experiment. Both the capillary force and the van der Waals force contribute to the adhesion force have been analysed in detail. We found that the triple point and the Kelvin radius that determined by the probe profile are the key parameters that play a decisive role in the adhesion force.

\section{Theory}

In Atomic Force Microscope (AFM) micro-handling manipulation, the liquid bridge formed by the water condensation serves as a channel between the probe and the substrate in the AFM experiment (Piner et al., 1999). The capillary forces are written by (Fisher, 1926)

$$
F_{\text {cap }}=2 \pi \gamma x_{p} \sin \left(\beta+\theta_{p}\right)+\pi x_{p}^{2} \Delta p
$$

The first term corresponds to the surface tension force and the second denotes the capillary (Laplace) pressure force. Here $\gamma$ is the surface tension coefficient, $x_{p}$ is the coordinate of the solid-liquid-vapor contact line (triple point), $\theta_{p}$ is the contact angle of the probe surface, $\beta$ is the half-filling angle and $D$ is the particle-surface distance. Under thermodynamic equilibrium, the relationship between the Relative Humidity $(\mathrm{RH})$, the pressure difference $\Delta p$ and the Kelvin radius $r_{k}$ obeys the Kelvin equation (Yang et al., 2010).

In nanoscale, van der Waals forces become important at the distances below 10-15 nm and may at these distances start to dominate are interactions of different origin that have been observed at large separations. The van der Waals force $F_{v d w}$ in a single medium is given by

$$
F_{v d w}=-\frac{H}{6}\left[\frac{R-D}{D^{2}}+\frac{3 R+D}{(D+2 R)^{2}}\right]
$$

where $R$ is probe radius and $H$ is the Hamaker constant. To avoid damaging of the surface, the separation $D$ for most solid contacts must be kept at least several tens of angstroms. For a general probe shape, it is expected that the van der Waals force between the probe and substrate when the liquid bridge in the gap is given by (Xiao and Qian, 1999)

$$
F_{v d w}=F_{v d w}^{w a t e r}\left\{1-\frac{1}{\left[1+\frac{y(x)}{D}\right]^{2}}\right\}+F_{v d w}^{a i r}\left\{1-\frac{1}{\left[1+\frac{y(x)}{D}\right]^{2}}\right\}
$$

where $F_{v d w}^{\text {water }}$ and $F_{v d w}^{a i r}$ are van der Waals forces with water and air as the medium, respectively. They can be calculated by Eq. (2.2) with different $H, R$ and $D . r_{k}, D$ and $\beta$ are related by

$$
r_{k}=\frac{x-r_{p s}\left[1-\cos \left(\beta+\theta_{p}\right)\right]}{\frac{x}{r_{p s}}-2+\sin \left(\beta+\theta_{p}\right)} \quad r_{p s}=\frac{D+y(x)}{\cos \left(\beta+\theta_{p}\right)+\cos \theta_{s}}
$$

where $r_{p s}$ is the liquid bridge meniscus radius and $\theta_{s}$ is the contact angle of the substrate.

For a spherical probe

$$
y(x)=\sqrt{R^{2}-x^{2}} \quad \beta=\arcsin \frac{x}{R}
$$

For parabolic probe, we adopt the power function relation

$$
y(x)=k x^{n} \quad \beta=\arctan y^{\prime}(x)
$$


The adhesion force is the sum of the capillary and van der Waals force (Lazzer et al., 1999). However, in most previous works (Jones et al., 2002; Junno et al., 1995), the van der Waals force is usually approximated or omitted in the calculation. In our simulation, we derive the analytical expression of the relation between the probe shape $r_{k}$ and $\beta$, Eqs. (2.4)-(2.6), and the adhesion force, which is the sum of the capillary and van der Waals force, is precisely calculated by using the probe geometry $y(x)$.

\section{Results and discussion}

In order to illustrate the necessity of the van der Waals force, we performed a comparison between the theoretical simulations and the experimental data for the $\mathrm{SiO}_{2}$ particle on a $\mathrm{TiO}_{2}$ surface, in which the adhesion force is calculated with and without the van der Waals force, respectively. When simulating without the van der Waals force, the pull-off force as a function of the RH is plotted in Fig. 1. The theoretical capillary force given by Eq. (2.1) using the parameters given by Paajanen et al. (2006). The simulation results showed that the capillary force is in a fairly good agreement with the experimental data at $\mathrm{RH} \geqslant 40 \%$, but deteriorates when $\mathrm{RH}<40 \%$. The large discrepancy between the theoretical results and the experiment data may be attributed to the fact that the adhesion force is dominated by the van der Waals force at a relatively low humidity.

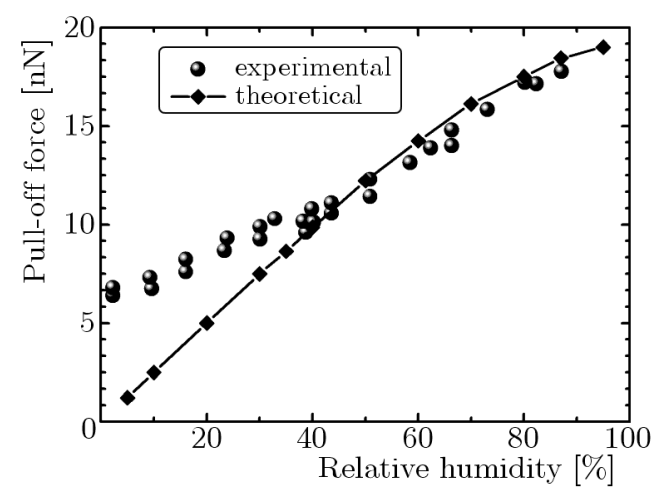

Fig. 1. The experimental data (Paajanen et al., 2006) and the theoretical pull-off force for a $\mathrm{SiO}_{2}$ particle on $\mathrm{TiO}_{2}$ surface versus the $\mathrm{RH} ; R=40 \mathrm{~nm}, \theta_{p}=0^{\circ}, \theta s=70^{\circ}, D=0.40 \mathrm{~nm}$

When the van der Waals force is included in simulation, the comparison of the theoretical results with the experiment data is shown in Fig. 2. It is indicated that the adhesion force between probe and substrate increases with the RH. In general, the simulation considering the van der Waals force provides a better description for the experimental data than that of considering the capillary force only. Although the van der Waals force is less dominant in the adhesion force, which is about several nano-Newtons to tens of nano-Newtons, it is should be taken into consideration. Therefore, the van der Waals force is contained in our following stimulation.

To clearly understand the influence of the tip shape on the adhesion force, we mainly investigate the adhesion force for the spherical probe and the parabolic probe on a substrate in the AFM experiment. The comparison between the experimental data and the stimulation results of the adhesion force for the parabolic probe as a function of $\mathrm{RH}$ is shown in Fig. 3. The adhesion force between the $\mathrm{Si}_{3} \mathrm{~N}_{4}$ probe and the $\mathrm{SiO}_{2}$ surface is employed to stimulate to explain the dependence of the adhesion force on the parabolic probe $\left(k=1.5 \cdot 10^{-4} \mathrm{~nm}^{-2}\right)$, which is the optimum function form of the probe shape for this experiment defined after many evaluations. In a general AFM experiment, the generic sketch of the functional relationship between the pull-off force and the Relative Humidity (RH) is inverted $U$-shaped (Xiao and Qian, 1999; Arai et al., 1996). The force increases steadily at low humidity, rises to a peak at intermediate humidity 


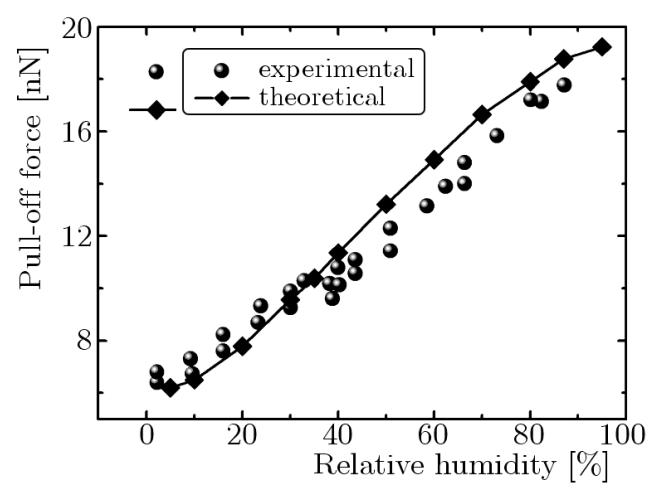

Fig. 2. Adhesion force for the $\mathrm{SiO}_{2}$ partice on the $\mathrm{TiO}_{2}$ surface as a function of $\mathrm{RH} ; R=40 \mathrm{~nm}$, $\theta_{p}=0^{\circ}, \theta_{s}=70^{\circ}, D=0.40 \mathrm{~nm}$ (Paajanen et al., 2006), $H=9.46 \cdot 10^{-20} \mathrm{~J}$ in air and $H=0.69 \cdot 10^{-20} \mathrm{~J}$ in water (Xiao and Qian, 1999)

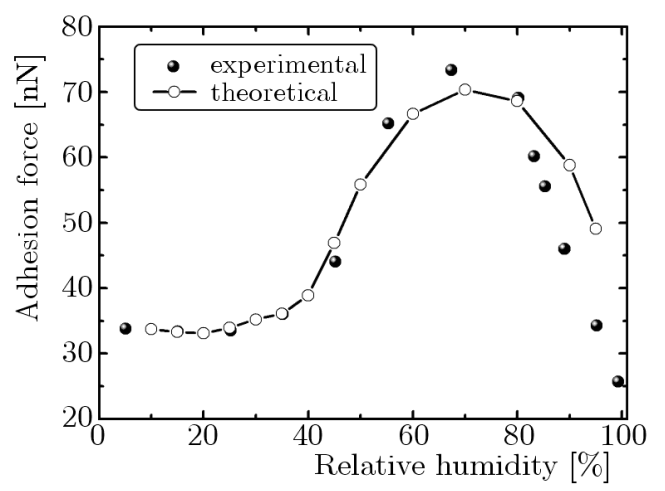

Fig. 3. The adhesion force between the $\mathrm{Si}_{3} \mathrm{~N}_{4}$ probe and the $\mathrm{SiO}_{2}$ surface as a function of $\mathrm{RH}$; $R=100 \mathrm{~nm}, \theta_{p}=60^{\circ}, \theta_{s}=0^{\circ}, D=0.8 \mathrm{~nm}$ (Xiao and Qian, 1999)

and falls in high humidity. The results predicted in our model show that the adhesion force grows with the increasing $\mathrm{RH}$, which is in accordance with the experiment data shown by Xiao and Qian (1999). The good results we obtained might be attributed to the inclusion of the Van der Waals force in low humidity. Some discrepancies appear at high humidity, possibly due to influence of the surface roughness which could reduce the Van der Waals force (Chen and Lin, 2008; Li et al., 2006; Sedin and Rowlen, 2000).

There are two different trends of the adhesion force in Figs. 2 and 3 with different probe shapes. The main acting force that guided the evolution of the adhesion force should be determined and carefully considered.

Figure 4 illustrates the spread of the capillary force and van der Waals force with RH. It is shown that the capillary force grows with an increase in the $\mathrm{RH}$ whereas the van der Waals force displays the contrary tendency. The reason is that the formation of the liquid bridge gradually reduces the van der Waals interaction between the particle and the surface (Lennart, 1997). Thus, either of these forces may become dominant depending on the humidity. At $\mathrm{RH} \leqslant 40 \%$, the capillary force and van der Waals force are in the same order magnitude. At $\mathrm{RH}>40 \%$, the bridge formed the gap gradually filling with water which reduces the van der Waals force from $5.1 \mathrm{nN}$ at a $\mathrm{RH}$ of $5 \%$ to $0.32 \mathrm{nN}$ at $87 \% \mathrm{RH}$. The capillary force interaction is very sensitive to the $\mathrm{RH}$, which is much larger than the van der Waals force under high $\mathrm{RH}$ conditions $(\mathrm{RH} \geqslant 40 \%)$. But the van der Waals force should not be omitted from the force equilibrium if the RH is lower than about $40 \%$. Our results are consistent with the previous experiment measurement (Christenson, 1988; Jang et al., 2006). 

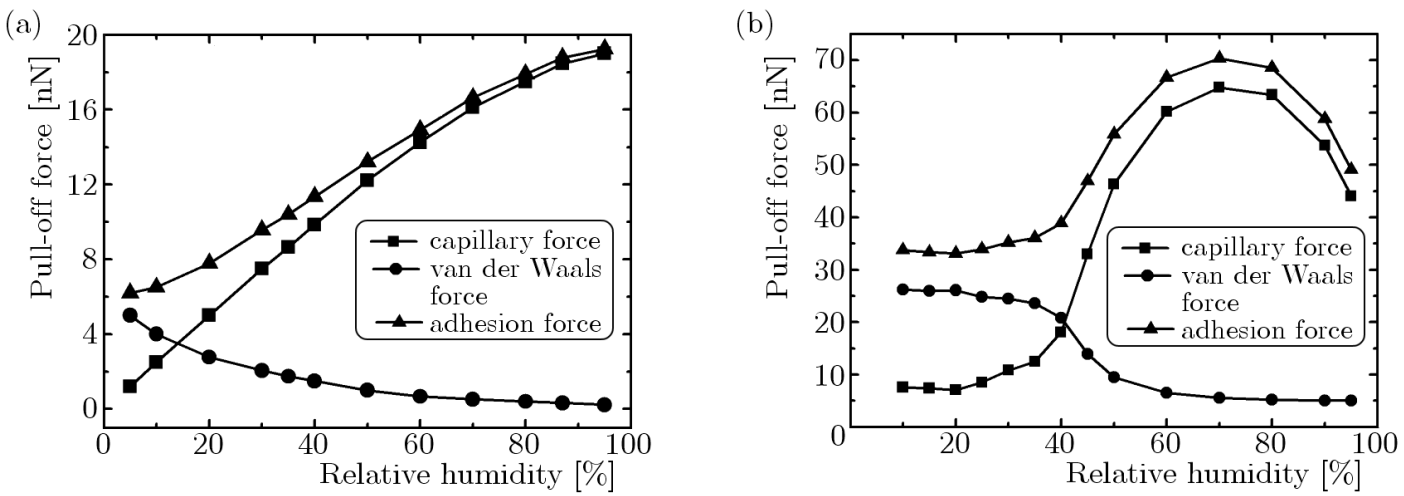

Fig. 4. The adhesion force together with the capillary force and van der Waals force varying with the RH; (a) spherical probe, (b) parabolic probe

From the previous investigation it can be found that the van der Waals force exhibits nonlinear trends with humidity. But it does not play a key role in the adhesion force variation, thus we put the emphasis on the capillary force. According to Eq. (2.1), the numerical magnitude of the capillary force is the mutual compensation of these two terms, the capillary (Laplace) pressure force and surface tension force (Aveyard et al., 1999). After numerical simulations, we found that the surface force linearly depends on humidity, while the capillary pressure force expresses fluctuating characters, which are similar to the adhesion force. The core of the adhesion force is the state parameter relation in the capillary pressure force.

Since the capillary (Laplace) force term predominated in the capillary force, whereas the $x_{p}$ and $r_{k}$, are the important factors in the capillary force according to Eq. (2.1), so two parameters are chosen to be analyzed in detail. The nonlinear variations of $x_{p}$ (triple point of the liquid bridge) and $r_{k}$ (Kelvin radius) with humidity for two different probe shapes are shown in Fig. 5. It can be seen that $x_{p}$ increases with humidity, whereas $r_{k}$ has the opposite trend. When the positive contribution to the capillary force from increasing $x_{p}$ is larger than the negative effect from decreasing $r_{k}$, the capillary force rises steadily, and vice versa. These two opposite contributions compete to determine the trend of the capillary force with humidity increment.
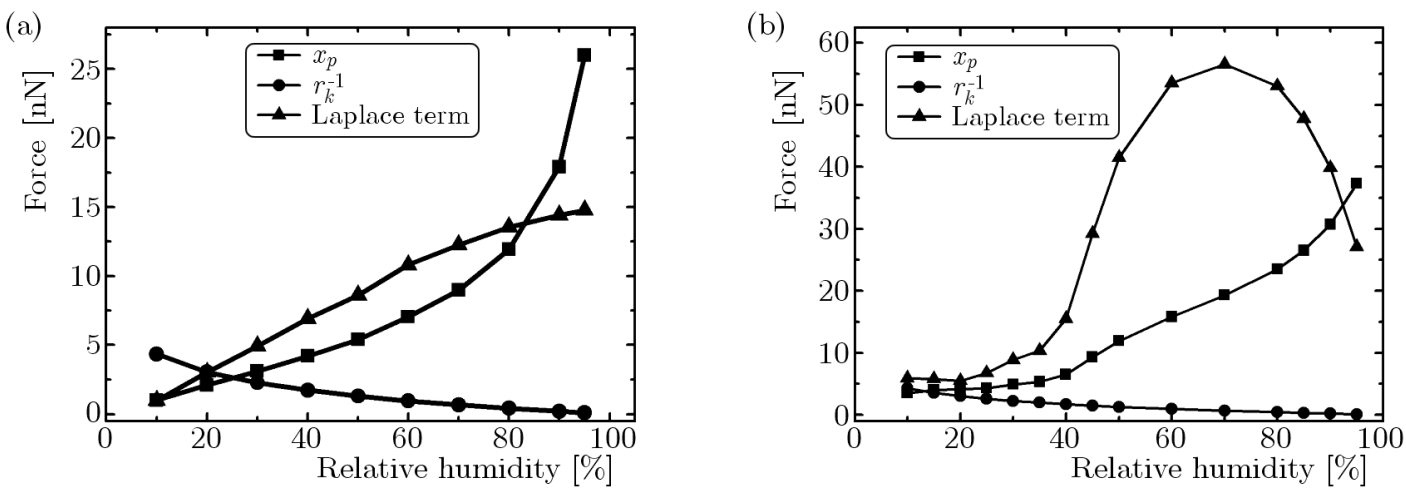

Fig. 5. Effects of $x_{p}$ and $r_{k}$ in the capillary pressure force; (a) spherical probe, (b) parabolic probe

At low humidity $(\mathrm{RH}<20 \%)$, the liquid bridge between the probe and substrate is very small and thin. Thus the value of $x_{p}$ is less than $r_{k}$, the total capillary force trend is decreasing. At the intermediate humidity $(40 \%<\mathrm{RH}<70 \%)$, the liquid bridge becomes wider and bigger. The increment of $x_{p}$ is larger than the $r_{k}$, so the total capillary force displays dramatic growth. At high humidity $(\mathrm{RH}>70 \%)$, the decrement of $r_{k}$ surpasses the increment of $x_{p}$, so the total capillary force starts to decrease. On the whole, the capillary force firstly decreases in low humidity, increases in intermediate humidity, and finally decreases at high humidity. Since the 
capillary force is the major determining force, we can see similar nonmonotonous behavior for the adhesion force in Fig. 4.

With the development of nano-science, materials science and surface science, various micromanipulation strategies have been proposed, such as electrostatic, vacuum, inertial handling. The use of the adhesion force is considered to be an effective, controllable and secure method in micro-manipulation. It is evident that the pickup manipulation benefits from the increase of the adhesion force, and the release manipulation benefits from the decrease of the adhesion force. Hence a wider adhesion force range induces better controllability during the AFM manipulation. Comparing to the spherical probe, the parabolic probe can give a larger interval between the smallest and largest values of the adhesion force, which is in a suitable range for practical operation and would be utilized to improve the handling efficiency.

\section{Conclusion}

In summary, we have analysed spherical and parabolic probes to simulate the effect of the probe shape on the adhesion force in the AFM operation. In order to clarify the correlation between the adhesion force and probe geometry, the key parameters that affect the adhesion force have been investigated through comparing the simulation results with the experiment data. The numerical simulation shows that the joint action of the triple point and the Kelvin radius are the main factors influencing the adhesion force variation, which are directly related to the probe shape in the ambient air.

\section{Acknowledgment}

This work has been supported by the National Natural Science Foundation of China under contract Nos. 11165004, 81060307, 11164002.

\section{References}

1. Arai F., Andou D., Fukuda T., 1996, Adhesion forces reduction for micro manipulation based on micro physics, Procedings of IEEE 9th Annual International Workshop on Micro Electro Mechanical Systems, 354-359

2. Aveyard R., Clint J.H., Paunov V.N., Nees D., 1999, Capillary condensation of vapours between two solid surfaces:effects of line tension and surface forces, Physical Chemistry Chemical Physics, 1, 155-163

3. Binnig G., Quate C.F., 1994, Atomic force microscope, Physical Review Letters, 56, 930-933

4. Butt H.J., Kappl M., 2009, Normal capillary forces, Advances in Colloid and Interface Science, 146, $48-60$

5. Chen S.C., Lin J.F., 2008, Detailed modeling of the adhesion force between an AFM tip and a smooth flat surface under different humidity levels, Journal of Micromechanics and Microengineering, 18, 115006-115013

6. Chen S.H., Soh A.K., 2008, The capillary force in micro and nano indentation with different indenter shapes, International journal of Solids and Structures, 45, 3122-3137

7. Christenson H.K., 1988, Adhesion between surfaces in undersaturated vapors- a reexamination of the influence of meniscus curvature and surface forces, Journal of Colloid Interface Science, 121, $1,170-178$

8. Eastman T., Zhu D.M., 1996, Adhesion forces between surface modified AFM tips and a mica surface, Langmuir, 12, 111, 2859-2862 
9. Fisher R.A., 1926, On the capillary forces in an ideal soil; correction of formulae given by W.B. Haines, Journal of Agricultural Science, 16, 492-505

10. Jang J., Ratner M.A., Schatz G.C., 2006, Atomic-scale roughness effect on capillary force in atomic force microscopy, Journal of Physics Chemstry B, 110, 659-662

11. Jones R., Pollock H.M., Cleaver J.A.S., Hodges C.S., 2002, Adhesion forces between glass and silicon surfaces in air studied by AFM: Effects of relative humidity, particle size, roughness, and surface treatment, Langmuir, 18, 21, 8045-8055

12. Junno T., Deppert K., Montelius L., Samuelson L., 1995, Controlled manipulation of nanoparticles with an atomic force microscope, Applied Physics Letters, 66, 26, 3627-3629

13. Lazzer A.D., Dreyer M., Rath H.J., 1999, Particle-surface capillary forces, Langmuir, 15, 4551-4559

14. LennART B., 1997, Hamaker constants of inorganic materials, Advances in Colloid and Interface Science, 18, 125-169

15. Li Q., Rudolph V., Peukert W., 2006, London-van der Waals adhesiveness of rough particles, Powder Technology, 161, 248-255

16. Paajanen M., Katainen J., Pakarinen O.H., Foster A.S., 2006, Experiment humidity dependency of small particle adhesion on silica and titania, Journal of Colloid and Interface Science, 304, 518-523

17. Piner R.D., Zhu J., Xu F., Hong S.H., Mirkin C.A., 1999, "Dip-Pen” nanolithography, Science, 283, 661-663

18. Quyang Q., Ishida K., OKADA K., 2001, Investigation of micro adhesion by atomic force microscopy, Applied Surface Science, 169, 644-648

19. Sedin D.L., Rowlen K.L., 2000, Adhesion forces measured by atomic force microscopy in humid air, Analytical Chemistry, 72910, 2183-2189

20. TAbrizi M.F., Kappl M., Butt H.J., 2008, Influence of humidity on adhesion: an atomic force microscope study, Journal of Adhesion Science and Technology, 22, 181-203

21. Tabrizi M.F., Kappl M., Cheng Y., Gutmann J., Butt H.J., 2006, On the adhesion between fine particles and nancontacts: An atomic force microscope study, Langmuir, 22, 2171-2184

22. Thomas R.C., Houston J.E., Crooks R.M., Kim T.S., Michalske T.A., 1995, Probing adhesion forces at the molecular scale, Journal of the American Chemical Society, 117, 13, $3830-3834$

23. Thundat T., Zheng X.Y., Chen G.Y., Sharp S.L., Warmack R.J., Schowalter L.J., 1993a, Characterization of atomic force microscope tips by adhesion force measurements, Applied Physics Letters, 63, 15, 2150-2152

24. Thundat T., Zheng X.Y., Chen G.Y., Warmack R.J., 1993b, Role of relative humidity in atomic force microscopy imaging, Surface Science Letters, 294, 939-943

25. Werf V.D., Putman K.O., Constant A.J., De G., Bart G., Greve J., 1994, Adhesion force imaging in air and liquid by adhesion mode atomic force microscopy, Applied Physics Letters, 65, 9, 1195-1197

26. Xiao X., QIAn L., 1999, Investigation of humidity-dependent capillary force, Langmuir, 16, 21, 8153-8158

27. YAng L., Tu Y.S., FAng H.P., 2010, Modeling the rupture of a capillary liquid bridge between a sphere and plane, Journal of Soft Matter, 6, 6178-6182 